\title{
Esterco bovino como substrato alternativo na produção de mudas de melão ${ }^{1}$
}

\author{
Vilmar Eurípedes da Silva Júnior ${ }^{2}$, Eduardo Pradi Vendruscolo ${ }^{3}$, Leandra Regina Semensato ${ }^{4}$, Luiz \\ Fernandes Cardoso Campos ${ }^{5}$, Alexsander Seleguini ${ }^{6}$
}

\begin{abstract}
Resumo: Alternativas aos métodos convencionais de produção de mudas devem ser buscados a todo momento, visando o aumento da qualidade e o retorno ao produtor. Nesse contexto, os resíduos da atividade pecuária, podem constar como opções ao agricultor. O objetivo deste trabalho foi avaliar o uso do esterco bovino como substrato alternativo em combinação com substrato comercial para a produção de mudas de melão. Para a composição dos experimentos foram semeadas duas variedades de melão (Cantaloupe e Amarelo) em bandejas de polietileno de 128 células, com cinco diferentes substratos: $100 \%$ esterco, $100 \%$ substrato comercial e três misturas de esterco bovino (EB) e substrato comercial (SC) $(75 / 25 ; 50 / 50 ; 25 / 75$, respectivamente), compondo os cinco tratamentos, com quatro repetições em blocos casualisados. Para o melão Cantaloupe, o esterco bovino curtido proporcionou incrementos significativos nas características biométricas das mudas, elevados teores das clorofilas e massa seca de parte aérea e raiz; o tratamento $75 \% \mathrm{~EB}+25 \% \mathrm{SB}$ apresentou resultados semelhantes para os teores de clorofilas, altura de planta, número de folhas, comprimento de raiz e índice de qualidade de Dickson. Para o melão Amarelo, o esterco bovino não apresentou os mesmos resultados, não ocorrendo diferença entre os tratamentos para os resultados de Clorofilas a e total, altura de planta, número de folhas e comprimento de raiz. O esterco bovino curtido possui potencial para substituir parcialmente ou integralmente o substrato comercial na produção de mudas de meloeiro.
\end{abstract}

Palavras-Chave: Cucumis melo L.; Nutrição de mudas; Melão rendilhado; Melão amarelo.

\section{Cattle manure as an alternative substrate in melon seedlings production}

\begin{abstract}
Alternatives to conventional methods of seedling production should be sought, aiming to increase quality and return to the producer. In this context, the residues of livestock activity may appear as options to the farmer. The objective of this work was to evaluate the use of cattle manure as an alternative substrate in combination with commercial substrate for the production of melon seedlings. For the composition of the experiments, two varieties of melon (Cantaloupe and Amarelo) were sowed in polyethylene trays of 128 cells, with five different substrates: $100 \%$ cattle manure, $100 \%$ commercial substrate and three mixtures of bovine manure (EB) and commercial substrate (SC) $(75 / 25,50 / 50,25 / 75$, respectively), composing the five treatments, with four replicates in randomized blocks. For Cantaloupe melon, cattle manure provided significant increases in the biometric characteristics of the seedlings, high contents of chlorophylls and dry mass of shoot and root; the treatment $75 \% \mathrm{~EB}+25 \% \mathrm{SB}$ presented similar results for the levels of chlorophylls, plant height, leaf number, root length and Dickson quality index. For Amarelo melon, bovine manure did not present the same results, with no difference between treatments for Chlorophyll a and total, plant height, leaf number and root length. The tanned cattle manure has the potential to partially or completely replace the commercial substrate in the production of melon seedlings.
\end{abstract}

Keywords: Cucumis melo L.; Seedling nutrition; Muskmelon; Yellow melon.

\footnotetext{
${ }^{1}$ Submetido em 20/11/2017 e aprovado em 06/05/2018

${ }^{2}$ Graduando em Agronomia, Uni-Anhanguera Centro Universitário de Goiás, Goiânia - Goiás, CEP: 74.423-115; E-mail: vilmarjr.agronomia@gmail.com

${ }^{3}$ Doutor em Agronomia; Bolsista PNPD, Universidade Estadual de Mato Grosso do Sul (UEMS), Unidade de Cassilândia, Programa de PósGraduação em Agronomia, Cassilândia - Mato Grosso do Sul, CEP: 79.540-000; E-mail: agrovendruscolo@ gmail.com

${ }^{4}$ Doutor em Agronomia; Docente, Universidade Estadual de Goiás (UEG), Campus de São Luís dos Montes Belos, São Luís dos Montes Belos - Goiás, CEP: 76.100-000; E-mail: luizfernandescampos@hotmail.com

${ }^{5}$ Doutora em Agronomia; Docente, Uni-Anhanguera Centro Universitário de Goiás, Goiânia - Goiás, CEP: 74.423-115; E-mail: ledocepe@gmail.com

${ }^{6}$ Doutor em Agronomia; Docente, Universidade Federal do Triângulo Mineiro, Campus Universitário de Iturama, Iturama - Minas Gerais, CEP: 38.280-000; E-mail: aseleguini@gmail.com
}

Revista Agropecuária Técnica, Areia-PB, v. 39, n. 2, p. 112-119, 2018

DOI: https://doi.org/ 10.25066/agrotec.v39i2.37234 


\section{Introdução}

O melão (Cucumis melo L) é uma cucurbitácea pertencente ao gênero Cucumis, o qual é composto por vários grupos e vêm ganhando, a cada ano, espaço no mercado. A produção brasileira de 2000 para 2015 , teve um aumento de $198,55 \%$, mais de 345 mil toneladas anuais. Em 2015 em pouco mais de 20 mil hectares de lavouras temporárias foram gerados cerca de 470 mil reais com a produção de melões, sendo o nordeste a região com maior produção, 94,72\%, principalmente nos estados do Rio Grande do Norte e Ceará, que juntos detêm 77,5\% dessa produção (IBGE/SIDRA, 2015). Para Silva (2012) o melão tem grande importância socioeconômica nas regiões produtoras levando uma oportunidade de melhoria na qualidade social das famílias que dependam da agricultura.

Em função do custo elevado das sementes, grande parte dos produtores opta pelo plantio através de mudas e não a semeadura direta como em outras culturas. Neste sentido, a obtenção de mudas de qualidade superior torna-se uma etapa de extrema importância por se tratar da fase inicial da lavoura, afetando significativamente o desenvolvimento vegetativo e a produção final (Campanharo et al., 2006; Hoffman et al., 2005).

Em complemento às tecnologias utilizadas para a manutenção da qualidade das mudas frente aos estresses ambientais, os substratos constam como fator de alta influência sobre a capacidade de desenvolvimento das plantas. Como descrevem Hoffman et al. (2005), substratos são materiais inertes utilizados como suporte físico. Os substratos enriquecidos proporcionam além de suporte, suprimento nutricional para o cultivo de mudas até o momento do transplante para o local de produção. Porém, a utilização em grande escala de substratos comerciais pode ser um fator que contribui para a oneração do processo produtivo de mudas (Vendruscolo et al., 2017), seja de viveirista ou do próprio produtor, caso ele produza suas próprias mudas.

Visando a redução dos custos de produção de olerícolas, Moreira et al. (2010) ressaltam o potencial uso de materiais alternativos, como resíduos orgânicos regionais de outros setores da produção na composição de substratos, propiciando a redução dos custos e destinando corretamente resíduos que possivelmente seriam desprezados. Materiais de origem vegetal, como fibra de coco, casca de arroz carbonizada, mucilagem de sisal e bagaço de cana-de-açúcar, dentre outros, têm-se destacado para uso na produção de mudas devido a aspectos considerados essenciais. Dentre esses aspectos estão a composição nutricional, alta disponibilidade e baixo custo de aquisição. Porém, é evidente a dificuldade para utilização isolada desses materiais como substrato para produção de mudas, por apresentarem, em sua composição, pelo menos um nutriente em deficiência (Severino; Lima; Beltrão, 2006; Ramos et al., 2012; Klein, 2015).

Dentre os resíduos produzidos pelo setor agropecuário, observa-se que o uso potencial do esterco na composição de substratos voltados ao desenvolvimento inicial de plantas está associado ao maior acúmulo de matéria seca, incremento das características biométricas (Gonçalves et al., 2014; Moura et al., 2015) e aumento dos teores relativos de clorofila (Silva et al., 2016). No entanto, novos estudos devem ser realizados com o intuito de estipularem-se adequações às diferentes espécies de interesse comercial, determinado o correto manejo e visando a obtenção de mudas com características adequadas de desenvolvimento.

Desta maneira, o objetivo deste trabalho foi de avaliar a eficiência de combinações entre esterco bovino curtido e substrato comercial na produção de mudas de duas variedades de meloeiros.

\section{Material e Métodos}

Dois experimentos foram conduzidos em telado localizado na Universidade Federal de Goiás, Goiânia - GO. A estrutura apresentava pé direito de quatro metros de altura e dimensões de $8 \mathrm{~m} \times 8 \mathrm{~m}$ (comprimento $\mathrm{x}$ largura), com cobertura e laterais utilizando tela de sombreamento de $50 \%$. Para a composição dos experimentos foram semeadas duas variedades de melão: Cantaloupe, cv. Trinity $(C)$ e Amarelo, cv. Ouro (A), realizada em bandejas de polietileno de 128 células preenchidas com cinco diferentes substratos (100\% esterco; $100 \%$ substrato) e três misturas de esterco bovino e substrato, $(75 / 25$; 50/50; 25/75), compondo os cinco tratamentos. O delineamento experimental foi em blocos casualizados, com quatro repetições, sendo que cada parcela foi composta por cinco plantas. Em cada célula foram semeadas duas sementes, procedendo-se a retirada de uma plântula logo após as contagens iniciais. 
Foram realizadas análises quanto à porosidade, densidade e fertilidade das diferentes combinações de substratos de acordo com as metodologias estabelecidas pela Empresa Brasileira de Pesquisa Agropecuária (Donagemma, 2011) (Tabela 1).

Realizaram-se contagens diárias da emergência de plântulas, dos cinco dias após semeadura até o fim da emergência das plântulas a fim de estabelecer o tempo médio de emergência (TME), conforme estabelecido por Labouriau (1983); o índice de velocidade de emergência (IVE), de acordo com a fórmula de Maguire (1962); e a porcentagem final de plântulas emergidas (PFE); com a estabilização da emergência das plântulas realizou-se o desbaste, deixando-se apenas uma plântula por célula.

Tabela 1 Análise físico-química dos substratos

\begin{tabular}{|c|c|c|c|c|c|c|c|c|}
\hline Substratos & $\begin{array}{c}\text { pH } \\
-\end{array}$ & $\mathbf{N}$ & $\mathbf{P}$ & $\mathbf{K}$ & $\begin{array}{c}\mathbf{C a} \\
\mathrm{g} \mathrm{kg}^{-1}\end{array}$ & Mg & MO & $\mathbf{C}$ \\
\hline $100 \% \mathrm{~EB}$ & 7,20 & 82,00 & 3,10 & 20,80 & 3,00 & 3,00 & 88,00 & 50,00 \\
\hline $75 \% \mathrm{~EB}+25 \% \mathrm{SC}$ & 6,85 & 66,00 & 2,70 & 18,90 & 3,30 & 3,00 & 86,80 & 49,30 \\
\hline $50 \% \mathrm{~EB}+50 \% \mathrm{SC}$ & 6,50 & 50,00 & 2,30 & 16,90 & 3,50 & 3,00 & 85,50 & 48,60 \\
\hline $25 \% \mathrm{~EB}+75 \% \mathrm{SC}$ & 6,15 & 34,00 & 1,90 & 15,00 & 3,80 & 3,00 & 84,30 & 47,90 \\
\hline $100 \% \mathrm{SC}$ & 5,80 & 18,00 & 1,40 & 13,00 & 4,00 & 3,00 & 83,00 & 47,20 \\
\hline \multirow{2}{*}{ Substratos } & \multirow[t]{2}{*}{$\mathbf{F e}$} & \multirow{2}{*}{\multicolumn{2}{|c|}{$\begin{array}{l}\text { Mn } \\
\ldots \ldots . m\end{array}$}} & $\overline{Z n}$ & \multirow{2}{*}{\multicolumn{2}{|c|}{$\mathbf{C} / \mathbf{N}$}} & \multirow{2}{*}{$\begin{array}{c}\text { PT } \\
\%\end{array}$} & $\overline{\text { DS }}$ \\
\hline & & & & & & & & $\mathrm{g} \mathrm{cm}^{3}$ \\
\hline $100 \% \mathrm{~EB}$ & 0,19 & \multicolumn{2}{|c|}{36,00} & 26,50 & \multicolumn{2}{|c|}{0,60} & 67,37 & 0,16 \\
\hline $75 \% \mathrm{~EB}+25 \% \mathrm{SC}$ & \multirow{2}{*}{$\begin{array}{c}66,14 \\
13209\end{array}$} & \multicolumn{2}{|c|}{48,25} & 27,10 & \multicolumn{2}{|c|}{0,75} & 62,98 & 0,19 \\
\hline $50 \% \mathrm{~EB}+50 \% \mathrm{SC}$ & & \multicolumn{2}{|c|}{60,50} & 27,70 & \multicolumn{2}{|c|}{0,97} & 65,08 & 0,22 \\
\hline $25 \% \mathrm{~EB}+75 \% \mathrm{SC}$ & 198,05 & \multicolumn{2}{|c|}{72,75} & 28,30 & \multicolumn{2}{|c|}{1,41} & 60,93 & 0,21 \\
\hline $100 \% \mathrm{SC}$ & 264,00 & \multicolumn{2}{|c|}{85,00} & 28,90 & \multicolumn{2}{|c|}{2,62} & 61,52 & 0,24 \\
\hline
\end{tabular}

$\mathrm{EB}=$ Esterco Bovino; $\mathrm{SC}=$ Substrato Comercial

Aos 30 dias após a semeadura, foram obtidos os teores relativos das clorofilas $\mathrm{a}, \mathrm{b}$ e total através do clorofilômetro digital CFL-1030; características biométricas das mudas: comprimento da parte aérea (CPA), pela medição com régua graduada desde o colo da planta até a inserção da última folha, comprimento de Raiz $(\mathrm{CR})$, pela medição com régua graduada do colo até o término da raiz, diâmetro do caule (DC), medido com paquímetro digital, número de folhas (NF) contabilizando folhas verdadeiras e expandidas que foram utilizadas para obtenção da área foliar (AF), por meio de leitura no software Easyleaf (Easlon; Bloom, 2014), e também as massas secas de parte aérea, raiz e total (MSPA, MSR, E MST), obtidas após a secagem em estufa com circulação de ar a $60^{\circ} \mathrm{C}$, até a constatação de massa constante, sendo os resultados de ambas, expressos em g plântula ${ }^{-1}$.

Determinou-se o índice de qualidade de Dickson (IQD) e o cálculo da relação raiz e parte aérea (R/PA), utilizando-se as massas secas das mudas na equação (Dickson et al., 1960):

$$
\mathrm{IQD}=\frac{\mathrm{MST}}{\left(\frac{\mathrm{CPA}}{\mathrm{DC}}\right)+\left(\frac{\mathrm{MSPA}}{\mathrm{MSR}}\right)}
$$

Os dados foram submetidos à análise de variância e as médias das variáveis submetidas ao teste de Tukey a $5 \%$ de probabilidade.

\section{Resultados e Discussão}

Para as variáveis ligadas à emergência das plântulas, não foram observadas diferenças significativas entre os tratamentos avaliados (Tabela 2). Em média, para o meloeiro Cantaloupe, as variáveis taxa de emergência, tempo médio de emergência e índice de velocidade de emergência foram de 88,44\%, 6,32 dias e 2,28 , respectivamente, enquanto que para o meloeiro Amarelo foram observadas médias de $68,44 \%$, 6,74 dias e 1,68, para essas mesmas características. Assim, pôde-se inferir que os tratamentos utilizados no presente estudo não interferiram sobre o desenvolvimento inicial das plantas, tanto para aquelas com maior vigor (Cantaloupe), quanto para as de menor vigor (Amarelo). Do ponto de vista econômico, a não interferência sobre esses fatores, indica que o substrato comercial pode ser substituído parcialmente ou integralmente pelo esterco, um resíduo em muitas propriedades rurais. 
Esses resultados não corroboram com os obtidos por Pereira et. al. (2012), que ao analisarem o esterco caprino e bovino na formação de substratos para a produção de mudas de melão, identificaram que o esterco bovino comprometeu todos os índices de emergência das plântulas em aproximadamente $50 \%$ quando comparado com o esterco caprino. Os autores relacionaram os efeitos negativos ao fato de que geralmente $o$ esterco caprino apresenta concentrações de NPK superiores ao esterco de bovinos, o que pode variar de animal para animal, principalmente em função da alimentação dos mesmos. Os malefícios causados pela utilização de compostos orgânicos também são relacionados a falta de um tratamento prévio, como a compostagem. Isso previne contra características indesejadas como a alta acidez e a salinidade (Gomes et al., 2008).

Tabela 2 Valores médios de taxa de emergência (TXE), tempo médio de emergência (TME) e índice de velocidade de emergência (IVE) de mudas de meloeiros cultivadas em diferentes substratos

\begin{tabular}{lcccccc}
\hline \multirow{2}{*}{ Substratos } & \multicolumn{3}{c}{ Cantaloupe } & \multicolumn{3}{c}{ Amarelo } \\
\cline { 2 - 6 } & TXE (\%) & TME (dias) & IVE & TXE (\%) & TME (dias) & IVE- \\
\hline $100 \%$ EB & $90,63 \mathrm{a}^{*}$ & $6,09 \mathrm{a}$ & $2,42 \mathrm{a}$ & $70,31 \mathrm{a}$ & $6,78 \mathrm{a}$ & $1,74 \mathrm{a}$ \\
$75 \% \mathrm{~EB}+25 \% \mathrm{SC}$ & $84,38 \mathrm{a}$ & $6,41 \mathrm{a}$ & $2,15 \mathrm{a}$ & $65,63 \mathrm{a}$ & $6,85 \mathrm{a}$ & $1,59 \mathrm{a}$ \\
$50 \% \mathrm{~EB}+50 \% \mathrm{SC}$ & $90,63 \mathrm{a}$ & $6,35 \mathrm{a}$ & $2,31 \mathrm{a}$ & $67,19 \mathrm{a}$ & $6,73 \mathrm{a}$ & $1,67 \mathrm{a}$ \\
$25 \%$ EB + 75\% SC & $84,38 \mathrm{a}$ & $6,40 \mathrm{a}$ & $2,16 \mathrm{a}$ & $71,88 \mathrm{a}$ & $6,73 \mathrm{a}$ & $1,76 \mathrm{a}$ \\
$100 \%$ SCO & $92,19 \mathrm{a}$ & $6,39 \mathrm{a}$ & $2,38 \mathrm{a}$ & $67,19 \mathrm{a}$ & $6,60 \mathrm{a}$ & $1,66 \mathrm{a}$ \\
\hline DMS & 16,77 & 0,91 & 0,47 & 21,25 & 1,17 & 0,41 \\
\hline
\end{tabular}

*Médias seguidas de mesma letra nas colunas não diferem entre si pelo teste de Tukey a 1\% ou 5\% de probabilidade. DMS

= Diferença mínima significativa, $\mathrm{EB}=$ Esterco Bovino, $\mathrm{SC}=$ Substrato Comercial.

Para os teores relativos de clorofila a, b e total observou-se que, o substrato composto pelo esterco bovino resultou em valores superiores quando utilizado na produção de mudas de meloeiro Cantaloupe, no entanto, diferindo significativamente apenas do substrato $50 \% \mathrm{~EB}+$
$50 \%$ SC para os teores relativos de clorofila a e total e do substrato $75 \% \mathrm{~EB}+25 \%$ SC para a clorofila b. Para o meloeiro Amarelo observouse diferença significativa apenas para os teores relativos de clorofila $b$, em que o substrato comercial propiciou maior valor (Tabela 3 ).

Tabela 3 Valores médios de teores relativos de clorofila a (CLA), b (CLB) e total (CLT), área foliar (AF) de mudas de meloeiros cultivadas em diferentes substratos

\begin{tabular}{|c|c|c|c|c|}
\hline \multirow{2}{*}{ Substratos } & \multicolumn{4}{|c|}{ Cantaloupe } \\
\hline & CLA (SPAD) & CLB & CLT & $\operatorname{AF}\left(\mathrm{cm}^{2}\right)$ \\
\hline $100 \% \mathrm{~EB}$ & $27,93 \mathrm{a}^{*}$ & $4,70 \mathrm{a}$ & $32,63 a$ & $9,76 a$ \\
\hline $75 \% \mathrm{~EB}+25 \% \mathrm{SC}$ & $26,69 \mathrm{ab}$ & $4,34 \mathrm{ab}$ & $31,03 \mathrm{ab}$ & $7,62 b$ \\
\hline $50 \% \mathrm{~EB}+50 \% \mathrm{SC}$ & $25,47 b$ & $4,18 b$ & $29,65 b$ & $5,81 \mathrm{c}$ \\
\hline $25 \% \mathrm{~EB}+75 \% \mathrm{SC}$ & $26,72 \mathrm{ab}$ & $4,02 b$ & $30,74 \mathrm{ab}$ & $4,89 \mathrm{c}$ \\
\hline $100 \% \mathrm{SCO}$ & $26,08 \mathrm{ab}$ & $4,09 \mathrm{~b}$ & $30,17 \mathrm{ab}$ & $3,43 \mathrm{~d}$ \\
\hline \multirow[t]{2}{*}{ DMS } & 2,12 & 0,51 & 2,48 & 1,40 \\
\hline & \multicolumn{4}{|c|}{ Amarelo } \\
\hline $100 \% \mathrm{~EB}$ & $26,88 \mathrm{a}$ & $4,12 b$ & $31,00 \mathrm{a}$ & $12,10 \mathrm{a}$ \\
\hline $75 \% \mathrm{EST}+25 \% \mathrm{SCO}$ & $24,21 \mathrm{a}$ & $3,61 b$ & $27,82 \mathrm{a}$ & $8,17 \mathrm{~b}$ \\
\hline $50 \% \mathrm{EST}+50 \% \mathrm{SCO}$ & $23,59 a$ & $3,54 b$ & $27,13 a$ & $6,65 b c$ \\
\hline $25 \% \mathrm{EST}+75 \% \mathrm{SCO}$ & $25,48 \mathrm{a}$ & $3,91 b$ & $29,38 \mathrm{a}$ & $5,05 \mathrm{~cd}$ \\
\hline $100 \% \mathrm{SCO}$ & $24,80 \mathrm{a}$ & $5,06 \mathrm{a}$ & $29,86 a$ & $3,10 \mathrm{~d}$ \\
\hline DMS & 3,43 & 0,61 & 3,93 & 2,08 \\
\hline
\end{tabular}

*Médias seguidas de mesma letra nas colunas não diferem entre si pelo teste de Tukey a 5\% de probabilidade. DMS = Diferença mínima significativa. $\mathrm{EB}=$ Esterco Bovino; $\mathrm{SC}=$ Substrato Comercial.

A pouca variação observada para os teores relativos de clorofilas entre os tratamentos é dada pela baixa demanda de nutrientes como o nitrogênio (Damasceno et al., 2011), o qual

Revista Agropecuária Técnica, Areia-PB, v. 39, n. 2, p. 112-119, 2018

DOI: https://doi.org/ 10.25066/agrotec.v39i2.37234 
participa das atividades fotossintetizantes e que afeta os teores relativos de clorofila em plantas adultas de melão (Damasceno et al., 2011) e melancia (Colla et al., 2011). As clorofilas atuam diretamente na captura de luz solar e a conversão desta em energia química, que é utilizada nos processos fisiológicas das plantas, sendo assim, estes pigmentos tem importante papel no desenvolvimento das plantas, atuando na fotossíntese, crescimento, e na capacidade de se adequarem a diferentes ambientes (Jesus; Marrenco, 2008).

Sendo a clorofila essencial aos processos fotoquímicos, sua redução no interior dos órgãos fotossintéticos resulta em diminuição dos processos relacionados a fixação de carbono, produção de energia e, consequentemente, afetará o desenvolvimento do vegetal (Taiz et al., 2017). Em complemento, Coelho et al. (2010) propõem que o nitrogênio pode ser estimado eficientemente através do teor relativo de clorofila foliar.

Para a área foliar, verificou-se que o esterco bovino propiciou maior desenvolvimento da área foliarem relação aos demais substratos, com as menores médias observadas para o substrato comercial (Tabela 3). A utilização do esterco bovino resultou em incrementos de $184,55 \%$ e 290,32\% desta variável para os meloeiros Cantaloupe e Amarelo, respectivamente, em relação à utilização do substrato comercial. Tais resultados assemelham-se aos encontrados por Oliveira et al. (2016) que avaliando desenvolvimento inicial da mamoneira sob diferentes fontes e doses de matéria orgânica, identificaram resposta linear, com a área foliar crescente com o incremento do teor de esterco bovino.

As folhas assumem funções muito relevantes na planta, como as de interceptar e absorver luz, realizar fotossíntese, trocas gasosas e transpiração (Taiz et al., 2017). Entende-se teoricamente que com uma maior área foliar, ocorra também uma maior taxa fotossintética, consequentemente um melhor desenvolvimento para as mudas (Pes; Arenhardt, 2015). Oliveira et al. (2016), constataram ao fertirrigar com diferentes concentrações de nutrientes, mudas de cultivares de maxixeiro em fibra de coco, que o índice de clorofila, juntamente com a área foliar, aumentaram linearmente em resposta ao aumento da concentração do nitrogênio, elemento que, como descrito anteriormente, encontra-se em quantidades superiores no esterco bovino em relação aos demais substratos.

Quanto à altura de plantas do melão Amarelo, o tratamento com $100 \%$ de SC foi o único a diferir estaticamente, apresentando a menor média. Para o número de folhas não ocorreu diferença significativa. No entanto, para o "Cantaloupe", houve diferença significativa para a altura de plantas entre os tratamentos de $100 \% \mathrm{~EB} ; 50 \%$ $\mathrm{EB}+50 \% \mathrm{SC}$ e $100 \% \mathrm{SB}$, enquanto que para o número de folhas $100 \% \mathrm{~EB}$ foi superior, não diferindo estatisticamente do tratamento $75 \%$ $\mathrm{EB}+25 \%$ SC (Tabela 4).

Tabela 4 Valores médios de teores relativos de altura de parte aérea (ALT), número de folhas (NF), comprimento de raiz (CR), massa seca de parte aérea (MSPA), raiz (MSR) e total (MST) e índice de qualidade de Dickson (IQD) de mudas de meloeiros cultivadas em diferentes substratos

\begin{tabular}{|c|c|c|c|c|c|c|c|c|}
\hline \multirow{2}{*}{ Substratos } & \multicolumn{8}{|c|}{ Cantaloupe } \\
\hline & ALT $(\mathrm{cm})$ & $\mathrm{NF}$ & $\mathrm{CR}(\mathrm{cm})$ & $\mathrm{DC}(\mathrm{mm})$ & MSPA $(g)$ & $\operatorname{MSR}(\mathrm{g})$ & $\operatorname{MST}(\mathrm{g})$ & IQD \\
\hline $100 \% \mathrm{~EB}$ & $5,45 a^{*}$ & $1,25 \mathrm{a}$ & $9,84 \mathrm{a}$ & $2,21 \mathrm{a}$ & $0,10 \mathrm{a}$ & $0,033 \mathrm{a}$ & $0,12 \mathrm{a}$ & $0,022 \mathrm{a}$ \\
\hline $75 \% \mathrm{~EB}+25 \% \mathrm{SC}$ & $5,00 \mathrm{ab}$ & $1,05 \mathrm{ab}$ & $9,57 \mathrm{ab}$ & $1,88 \mathrm{~b}$ & $0,07 \mathrm{~b}$ & $0,027 b$ & $0,10 \mathrm{~b}$ & $0,018 \mathrm{ab}$ \\
\hline $50 \% \mathrm{~EB}+50 \% \mathrm{SC}$ & $4,37 \mathrm{~b}$ & $1,00 \mathrm{~b}$ & $8,11 \mathrm{~b}$ & $1,84 \mathrm{~b}$ & $0,06 \mathrm{c}$ & $0,023 \mathrm{c}$ & $0,09 \mathrm{c}$ & $0,016 b$ \\
\hline $25 \% \mathrm{~EB}+75 \% \mathrm{SC}$ & $4,71 \mathrm{ab}$ & $1,00 \mathrm{~b}$ & $8,02 b$ & $1,79 b$ & $0,06 \mathrm{c}$ & $0,023 \mathrm{c}$ & $0,08 \mathrm{~cd}$ & $0,017 b$ \\
\hline $100 \% \mathrm{SCO}$ & $3,28 \mathrm{c}$ & $1,00 \mathrm{~b}$ & $9,07 \mathrm{ab}$ & $1,69 b$ & $0,05 \mathrm{~d}$ & $0,022 \mathrm{c}$ & $0,07 \mathrm{~d}$ & $0,018 \mathrm{ab}$ \\
\hline \multirow[t]{2}{*}{ DMS } & 0,77 & 0,21 & 1,70 & 2,00 & 0,01 & 0,004 & 0,01 & 0,004 \\
\hline & \multicolumn{8}{|c|}{ Amarelo } \\
\hline $100 \% \mathrm{~EB}$ & $7,16 a$ & $1,05 \mathrm{a}$ & $10,28 \mathrm{a}$ & $2,39 a$ & $0,11 \mathrm{a}$ & $0,030 \mathrm{a}$ & $0,14 \mathrm{a}$ & $0,020 \mathrm{a}$ \\
\hline $75 \% \mathrm{EST}+25 \% \mathrm{SCO}$ & $6,95 a$ & $1,00 \mathrm{a}$ & $9,41 \mathrm{a}$ & $2,21 \mathrm{a}$ & $0,08 b$ & $0,025 b$ & $0,10 \mathrm{~b}$ & $0,017 \mathrm{ab}$ \\
\hline $50 \% \mathrm{EST}+50 \% \mathrm{SCO}$ & $7,34 \mathrm{a}$ & $1,00 \mathrm{a}$ & $9,24 \mathrm{a}$ & $1,94 b$ & $0,06 \mathrm{~cd}$ & $0,025 b$ & $0,09 b$ & $0,014 b$ \\
\hline $25 \% \mathrm{EST}+75 \% \mathrm{SCO}$ & $6,73 a$ & $1,00 \mathrm{a}$ & $9,06 \mathrm{a}$ & $1,96 b$ & $0,07 b c$ & $0,025 b$ & $0,10 \mathrm{~b}$ & $0,014 b$ \\
\hline $100 \%$ SCO & $4,89 \mathrm{~b}$ & $1,00 \mathrm{a}$ & $9,04 \mathrm{a}$ & $1,97 \mathrm{~b}$ & $0,05 \mathrm{~d}$ & $0,030 \mathrm{a}$ & $0,09 \mathrm{~b}$ & $0,020 \mathrm{a}$ \\
\hline DMS & 0,85 & 0,09 & 1,44 & 0,23 & 0,01 & 0,001 & 0,02 & 0,004 \\
\hline
\end{tabular}

*Médias seguidas de mesma letra nas colunas não diferem entre si pelo teste de Tukey a 5\% de probabilidade. DMS = Diferença mínima significativa. $\mathrm{EB}=$ Esterco Bovino; $\mathrm{SC}=$ Substrato Comercial. 
Adequado desenvolvimento em altura da parte aérea e do número de folhas com uso do esterco bovino também puderam ser observados em mudas de melancia em diferentes substratos por Silva et al. (2009); o que foi relatado também por Mesquita et.al (2012) estudando a produção de mudas de mamoeiro com uso de substratos contendo o esterco bovino, identificaram que o número de folhas e altura da planta foram influenciados significativamente pela percentagem de esterco bovino na composição do substrato, apresentando um aumento contínuo com o aumento das concentrações de esterco, relacionando os resultados com o maior teor de fósforo, potássio, cálcio e magnésio disponíveis no esterco bovino, influenciando a emissão e o tamanho das folhas.

Em relação as variáveis diâmetro do caule e massa seca da parte aérea, o tratamento com $100 \%$ de esterco bovino foi o único a diferir significativa para o melão Cantaloupe, resultado que se repete para o melão Amarelo quanto aos valores de massa seca de parte aérea. No entanto, para o diâmetro do caule, o tratamento $75 \% \mathrm{~EB}+$ $25 \%$ SC também diferiu dos demais, porém $7,53 \%$ menor que o obtido no tratamento de $100 \%$ EB. Para massa seca total observou-se superioridade para o tratamento com $100 \%$ EB nos dois tipos de melão, sendo que em comparação ao tratamento com $100 \%$ SC, $100 \%$ EB foi superior em 41,7\% para o Cantaloupe e $35,71 \%$ para o Amarelo (Tabela 4).

De acordo com Souza et al. (2013), o diâmetro do caule e o comprimento da parte aérea, são dois dos mais importantes caracteres morfológicos para se estimar o crescimento das mudas após o plantio definitivo. Os maiores valores em massas secas da parte aérea e total podem ser explicados pelos teores de nutrientes encontrados no esterco bovino. Juntamente à atuação do nitrogênio, o magnésio é parte estrutural da molécula de clorofila; o fósforo compõe nucleotídeos usados no metabolismo energético das plantas, como o ATP, além do enxofre que compõe coenzimas e vitaminas, essenciais ao metabolismo (Taiz et al., 2017).

Para o Comprimento de raiz e massa seca de raiz não houve diferença significativa para $o$ melão Amarelo; já para o melão Cantaloupe, notou-se superioridade do tratamento com $100 \%$ esterco bovino, porém não diferiu estaticamente dos tratamentos com $75 \% \mathrm{~EB}+25 \%$ SC e $100 \%$ SC (Tabela 4), indicando um melhor desenvolvimento do sistema radicular, uma vez que este tratamento apresentou ainda melhores índices de porosidade total e menor densidade de partículas, aliados aos melhores níveis de fertilidade encontrados no esterco bovino (Tabela 1). Isso implicará que, ao ser transplantada, a muda possuirá capacidade de realizar adequadamente a absorção de elementos essenciais e garantir seu pleno desenvolvimento (Vendruscolo et al., 2016).

O IQD (Índice de Qualidade de Dickson) foi superior para os dois tipos de melão no tratamento com $100 \%$ EB, no entanto, em ambos os casos não diferiu significativamente dos tratamentos $75 \%$ EB $+25 \%$ SC e $100 \%$ SC (Tabela 4). Esse índice é um indicador da qualidade das mudas que considera no cálculo a massa seca total, o comprimento da parte aérea, o diâmetro do caule, a massa seca da parte aérea e massa seca da raiz, ou seja, reflete a robustez e o equilíbrio da distribuição da biomassa na muda; sendo assim ressalta os resultados de vários parâmetros de importância para avaliação da qualidade e como essa muda se desenvolverá no campo, após seu transplante (Fonseca et al., 2002).

\section{Conclusão}

O esterco bovino curtido possui potencial para substituir parcialmente ou integralmente $o$ substrato comercial na produção de mudas de meloeiro.

\section{Referências}

Campanharo, M.; Rodrigues, J. J. V; Lira Junior, M. D. A., Espindula, M. C.; Costa, J. V. T. Características físicas de diferentes substratos para produção de mudas de tomateiro. Revista Caatinga, v.19, n.2, p.140-145, 2006. http://www.redalyc.org/html/2371/237117566007l

Coelho, F. S.; Fontes, P. C. R.; Puiatti, M.; Neves, J. C. L.; Silva, M. C. de C. Dose de nitrogênio associada à produtividade de Batata e índices do estado de nitrogênio na folha. Revista Brasileira de Ciência do Solo, v.34, n.4, p.1175-1183, 2010. http://dx.doi.org/10.1590/S0100$\underline{06832010000400017}$

Colla, G.; Rouphael, Y.; Mirabelli, C.; Cardarelli, M. Nitrogen-use efficiency traits of miniwatermelon in response to grafting and 
nitrogen- fertilization doses. Journal of Plant Nutrition and Soil Science, v.174, n.6, p.933941, 2011. https://doi.org/10.1002/jpln.201000325

Damasceno, A. P. A. B.; Medeiros, J. F.; Medeiros, D. C.; Costa, I. G.; Dantas, D. C. Crescimento e marcha de absorção de nutrientes do melão Cantaloupe tipo "Harper" fertirrigado com doses de N e K. Revista Caatinga, v.25, n.1, p.137-146, 2011. http://www.redalyc.org/pdf/2371/237123860020.pdf

Dickson, A.; Leaf, A. L.; Hosner, J. F. Quality appraisal of white spruce and white pine seedling stock in nurseries. Forestry Chronicle, v.36, n.1, p.10-13, 1960. https://doi.org/10.5558/tfc36010-1

Donagemma, G. K.; Campos, D. B.; Calderano, S. B.; Teixeira, W. G.; Viana, J. M. Manual de métodos de análise de solo. 2.ed. Brasília: Embrapa, 2011.230p.

Easlon, H. M.; Bloom, A. J. Easy Leaf Area: Automated digital image analysis for rapid and accurate measurement of leaf area. Plant $\begin{array}{lllll}\text { sciences, } & \text { v.2, } & \text { n.7, } & \text { p.1-4, } 2014 .\end{array}$ https://doi.org/10.3732/apps.1400033

Fonseca, É. D. P.; Valéri, S. V.; Miglioranza, É.; Fonseca, N. A. N.; Couto, L. Padrão de qualidade de mudas de Trema micrantha (L.) Blume, produzidas sob diferentes períodos de sombreamento. Revista Árvore, v.26, n.4, p.515-523, 2002. http://www.scielo.br/pdf/\%0D/rarv/v26n4/a15v26n4.pdf

Gomes, L. A. A.; Rodrigues, A. C.; Collier, L. S.; Feitosa, S. D. S. Produção de mudas de alface em substrato alternativo com adubação. Horticultura Brasileira, v.26, n.3, p.359-363, $2008 . \quad$ https://doi.org/10.1590/S0102$\underline{05362008000300013}$

Gonçalves, E. O.; Petri, G. M.; Caldeira, M. V. W.; Dalmaso, T. T.; Silva, A. G. Crescimento de mudas de Ateleia glazioviana em substratos contendo diferentes materiais orgânicos. Floresta e Ambiente, v.21, n.3, p.339-348, 2014. https://doi.org/10.1590/2179-8087.029213

Hoffmann, A.; Nachtigal, J. C.; Fachinello, J. C. Infra-estrutura para propagação de plantas frutíferas. Brasília: Embrapa, 2005. 221p.

IBGE/SIDRA. Tabela 1612 - Área plantada, área colhida, quantidade produzida, rendimento médio e valor da produção das lavouras temporárias: banco de dados.
Disponível em: < http://sidra.ibge.gov.br/tabela/1612 >. Acesso em: 21 de abril 2017.

Jesus, S. V.; Marenco, R. A. O SPAD-502 como alternativa para a determinação dos teores de clorofila em espécies frutíferas. Acta Amazonica, v.38, n.4, p.815-818, 2008. https://dx.doi.org/10.1590/S0044-59672008000400029

Klein, C. Substratos Alternativos Para Produção De Mudas. Revista Brasileira de Energias Renováveis, v.4, n.3, p.43-63, 2015. http://dx.doi.org/10.5380/rber.v4i3.40742

Labouriau, L.G. A germinação de sementes. Washington, DC: Secretaria Geral da Organização dos Estados Americanos. 1983. 174p.

Maguire, J.D. Speed of germination-aid in selection and evaluation for seedlings emergence and vigor. Crop Science, v.2, n.2, p.176-177, 1962. https://dl.sciencesocieties.org/publications/cs/abstracts/2/ 2/CS0020020176

Mesquita, E. F.; Chaves, L. H.; Freitas, B. V.; Silva, G. A.; Sousa, M. V.; Andrade, R. Produção de mudas de mamoeiro em função de substratos contendo esterco bovino e volumes de recipientes. Revista Brasileira de Ciências Agrárias, v.7, n.1, p.58-65, 2012. http://www.redalyc.org/html/1190/119023656008/

Moreira, M. A.; Dantas, F. M.; Bianchini, F. G.; Viégas, P. R. A. Produção de mudas de berinjela com uso de pó de coco. Revista Brasileira de Produtos Agroindustriais, v.12, n.2, p.163170, 2010. http://www.deag.ufcg.edu.br/rbpa/rev122/Art1227.pdf

Moura, M. D. C. F.; Lima, L. K. S.; Rodrigues, K. K. R. P.; Santos, C. C.; Dutra, A. S. Utilização de substratos orgânicos para a produção de mudas de Amburana cearense. Nature and Conservation, v.8, n.1, p.6-12, 2015. https://doi.org/10.6008/SPC2318-2881.2015.001.0001

Oliveira, F. D. A.; Oliveira, M. K. T.; Martins, D. C.; Souza Neta, M. L.; Medeiros, J. F. Produção de mudas de cultivares de maxixeiro em fibra de coco fertiirrigadas com diferentes concentrações de nutrientes. Revista Ceres, v.63, n.5, p.698-705, 2016. https://doi.org/10.1590/0034-737X201663050015

Pereira, D. L.; Oliveira, R. H.; Souza, E. G. F.; Ferraz, A. P. F.; Coelho Junior, L. F.; Barros Junior, A. P. Uso de fontes orgânicas como 
substrato na produção de mudas de melão. Horticultura Brasileira, v.30, n.2, p.55995605 , 2012. http://www.abhorticultura.com.br/eventosx/trabalhos/ev 6/a5428_t8082_comp.pdf

Pes, L.S; Arenhardt.M.H. Fisiologia vegetal. Universidade Federal de Santa Maria, Colégio Politécnico, Rede e-Tec Santa Maria, RS, 81.p, 2015.

Ramos, A. R.; Dias, R. C. S.; Aragão, C. A.; Mendes, A. M. Mudas de melancia produzidas com substrato à base de pó de coco e soluções nutritivas. Horticultura Brasileira, v.30, n.2, p.339-344, 2012. https://doi.org/10.1590/S0102$\underline{05362012000200026}$

SEVERINO, L. S.; LIMA, R. L. S.; BELTRÃO, N. E. M. Composição Química de Onze Materiais Orgânicos Utilizados em Substratos para Produção de Mudas: Comunicado Técnico. n. 278. Campina Grande: Embrapa Algodão. 2006. https://ainfo.cnptia.embrapa.br/digital/bitstream/CNPA/1 8364/1/COMTEC278.pdf

Silva, A. C.; Cavalcante, A. C. P.; Cavalcante, A. G.; Neto, M. A. D. Bactérias fixadoras de nitrogênio e substratos orgânicos no crescimento e índices clorofiláticos de amendoim. Agropecuária Técnica, v.37, n.1, p.1-8,

2016. https://doi.org/10.25066/agrotec.v37i1.27964

Silva, E. C.; Costa, C. C.; Santana, J. B. L.; Monteiro, R. F.; Ferreira, E. F.; Silva, A. S. Avaliação de diferentes tipos de substratos na produção de mudas de melancia. Horticultura
Brasileira, v.27, S3142-S3146, 2009. http://www.abhorticultura.com.br/eventosx/trabalhos/ev 3/A2152_T3296_Comp.pdf

Silva. M. C. Melão rendilhado em ambiente protegido submetido à doses de nitrogênio $\mathrm{e}$ potássio em Rondonópolis-MT. 2012. 104 f. Dissertação (Mestrado em Engenharia de Sistemas Agrícolas) - Universidade Federal de Mato Grosso, Rondonópolis, 2012. http://www.ufmt.br/pgeagri/arquivos/25c4ee745121d2a2 328653440b96d708.pdf

Souza, E. G. F.; Barros Júnior, A. P.; Silveira, L. M.; Santos, M. G.; Silva, E. F. S. Emergência e desenvolvimento de mudas de tomate IPA 6 em substratos, contendo esterco ovino. Revista Ceres, v.60, n.6, p.902-907, 2013. http://www.redalyc.org/html/3052/305229913020/

Taiz, L.; Zeiger, E.; Moller, I. M.; Murphy, A. Fisiologia e desenvolvimento vegetal. 6 . ed. Artmed, Porto Alegre. 2017. 888 p.

Vendruscolo, E. P.; Martins, A. P. B.; Campos, L. F. C.; Brandão, D. C.; Nascimento, L. M.; Seleguini, A. Produção de mudas de batatadoce de baixo custo em diferentes substratos e níveis de enfolhamento de estacas. Revista de Agricultura Neotropical, v.4, n.2, p.102-110, 2017.

https://periodicosonline.uems.br/index.php/agrineo/article /view/1429/1414

Vendruscolo, E.P.; Martins, A.P.B.; Seleguini, A. Promoção no desenvolvimento de mudas olerícolas com uso de bioestimulante. Journal of gronomic Sciences, Umuarama, v.5, n.2, p.73-82, 2016. http://www.dca.uem.br/V5N2/08.pdf 